stimulants was started (filgrastim, $480 \mu \mathrm{g}$ intravenously, and sargramostim, $500 \mu \mathrm{g}$ intravenous piggyback every 12 hours). On POD 37, further debridement and reconstruction of the sternum with omental transfer and split-thickness skin grafting were done.

The remainder of the hospital course was marked by extensive surgical wound care, steroid therapy, intermittent dialysis, chemical bone marrow stimulation, nutritional support, and physical rehabilitation. The patient was transferred to a rehabilitation center on POD 72. At hospital discharge, the white blood cell count was 9.6 $\mathrm{K} / \mathrm{UL}$, hemoglobin and hematocrit values were $7.0 \mathrm{gm} / \mathrm{dl}$ and $21.6 \%$, and platelet count was $15 \mathrm{~K} / \mathrm{UL}$.

Discussion. Pyoderma gangrenosum is a rare disorder and only two case reports document the disease after coronary artery bypass grafting procedures. ${ }^{1,2}$ The cause of pyoderma gangrenosum is unknown. It is, however, immunologic in nature. ${ }^{3}$ Characteristically, the lesions are found after an injury to the skin. ${ }^{2}$ There are no organisms found on Gram stain tests and there is no growth on culture. Typically, there are ulcerations marked by well-defined borders with a blue outline indicating necrosis of the subcutaneous tissue. Although the diagnosis of pyoderma gangrenosum is clinical, there are characteristic histopathologic features: acute and chronic inflammatory changes, endothelial proliferation, loss of the epidermis at the ulcerated sites, and epidermal hyperplasia at the borders.

In our case and in another previously described, ${ }^{4}$ pyoderma gangrenosum was linked to myeloproliferative disease. In the report of Romano and Safai, ${ }^{4}$ the patient was known to have chronic myelocytic leukemia. In that case, the skin lesions responded to a course of oral prednisone beginning with $80 \mathrm{mg}$ per day. ${ }^{4}$ In our case, the disease was discovered during the evaluation of pancytopenia. This delay in diagnosis resulted in further skin and subcutaneous necrosis that necessitated extensive debridement. Once the diagnosis was made, steroid therapy arrested the necrotic process and reversed the cutaneous pathologic changes. In a similar case, cyclosporine A (5 $\mathrm{mg} / \mathrm{kg}$ ) was successfully used to avoid the wound-healing problems associated with steroid use. ${ }^{2}$ In our case, the use of cyclosporine was not an option because of the chronic renal failure and pancytopenia.

The challenging component of our case was the prevention of sepsis in the setting of pancytopenia with extensive open surgical wounds. Broad-spectrum antibiotic coverage, strict isolation, frequent surveillance cultures, and pharmacologic stimulation of the bone marrow were necessary. Despite these steps, various local infections occurred, including dialysis access-related infection and upper respiratory tract infection. Fortunately, systemic involvement was avoided.

In conclusion, concurrent pyoderma gangrenosum and myeloproliferative disease represents a unique and challenging clinical problem. Early recognition of the skin manifestations of pyoderma gangrenosum is necessary so that steroid therapy can be instituted, thereby avoiding radical debridement of wounds. The therapy for the associated pancytopenia requires support of the bone marrow with chemical stimulants. Prophylactic broad-spectrum antibiotic coverage is necessary to prevent sepsis.

\section{REFERENCES}

1. Rand RP, Olerud JE, Verrier ED. Pyoderma gangrenosum after coronary artery bypass grafting. Ann Thorac Surg 1993; 55:1016-8.

2. Koss-Harnes D, Ovrum E, Langeland T. Pyoderma gangrenosum as a complication of coronary artery bypass grafting. Eur J Cardiothorac Surg 1995;9:163-5.

3. Delescluse J, de Bast CL, Achten G. Pyoderma gangrenosum with altered cellular immunity and dermonecrotic factor. $\mathrm{Br} \mathbf{J}$ Dermatol 1972;82:529-32.

4. Romano J, Safai B. Pyoderma gangrenosum and myeloproliferative disorders. Arch Intern Med 1979;139:932-4.

\title{
EFFECTS OF POSTERIOR PERICARDIOTOMY ON THE INCIDENCE OF ATRIAL FIBRILLATION AND CHEST DRAINAGE AFTER CORONARY REVASCULARIZATION: A PROSPECTIVE RANDOMIZED TRIAL
}

\author{
George Asimakopoulos, MD, Renato Della Santa, MD, and David P. Taggart, MD(Hons), FRCS, \\ Oxford, United Kingdom
}

From the Department of Cardiothoracic Surgery, Oxford Heart Center, The John Radcliffe Hospita1, Oxford, United Kingdom.

Received for publication June 25, 1996; accepted for publication Sept. 11, 1996.

Address for reprints: D. P. Taggart, MD (Hons), FRCS, Consultant Cardiothoracic Surgeon, The John Radcliffe Hospital, Oxford OX3 9DU, United Kingdom.

J Thorac Cardiovasc Surg 1997;113:797-9

Copyright (C) 1997 by Mosby-Year Book, Inc.

$0022-5223 / 97 \$ 5.00+0 \quad \mathbf{1 2 / 5 4 / 7 7 8 6 3}$
Supraventricular tachyarrhythmia (SVT), mainly in the form of atrial fibrillation (AF), occurs in up to $40 \%$ of patients undergoing coronary artery bypass grafting (CABG) and although usually benign can cause hemodynamic instability, prolong hospital stay and increase costs, and, rarely, predispose to a cerebrovascular accident. ${ }^{1}$

Pericardial effusions are incriminated in the development of SVT after CABG. ${ }^{2}$ Posterior pericardiotomy has recently been reported to reduce the incidence of echocardiographically defined pericardial effusions from $40 \%$ in a control group to $8 \%$ in a pericardiotomy group with 
Table I. Demographic, preoperative, and intraoperative characteristics of the two groups

\begin{tabular}{lccc}
\hline & Control group & Pericardiotomy group & $p$ Value \\
\hline No. & 50 & 50 & NS \\
Mean age (yr) & $61(2)$ & $61(9)$ & NS \\
LV function & 32 & 38 & NS \\
$\quad$ Good & 12 & 10 & NS \\
Moderate & 6 & 2 & NS \\
$\quad$ Poor & 34 & 28 & NS \\
Preop. beta blockers & & 10 & NS \\
Risk factors & 5 & 25 & NS \\
$\quad$ Diabetes & 23 & 26 & NS \\
Hypercholesterolemia & 25 & 10 & NS \\
Smoking & 19 & $2.7(0.6)$ & \\
$\quad$ Hypertension & $2.7(0.7)$ & 48 & NS \\
No. of grafts & 47 & 22 & NS \\
Type of arterial grafts & 18 & 3 & NS \\
Left internal thoracic & 7 & $35(2)$ & NS \\
Radial & $33(8)$ & $66(17)$ & NS \\
Endarterectomy & $62(17)$ & & \\
Ischemic time (min) & & & \\
CPB time (min) & & & \\
\hline
\end{tabular}

Numbers in parentheses are the standard deviation. NS, Not significant; $C P B$, cardiopulmonary bypass.

Table II. Postoperative data in the two groups

\begin{tabular}{lccc}
\hline & Control group & Pericardiotomy group & $p$ Value \\
\hline No. & 50 & 50 & \\
Ventilation time (hr) & $7(4)$ & $7(7)$ & $<0.01$ \\
Blood loss (ml) & $660(238)$ & $831(303)$ & $<0.001$ \\
At $12 \mathrm{hr}$ & $813(282)$ & $1032(367)$ & \\
Total & & 12 & 1 \\
Arthythmias $(n)$ & 9 & 13 & 1 \\
AF & 1 & 1 & \\
Other & 10 & 1 & \\
Total & 1 & & \\
IABP & 1 & & \\
Death &
\end{tabular}

$L A B P$, Intraaortic balloon pump.

a simultaneous reduction in the incidence of SVT from $36 \%$ to $8 \% .^{3}$ That study was not, however, strictly randomized and neither the number of participating surgeons nor their method or methods of myocardial protection was specified.

In a prospective, randomized trial we tested the hypothesis that posterior pericardiotomy reduces the postoperative incidence of SVT by improving pericardial drainage.

Methods. One hundred consecutive patients undergoing isolated first-time CABG by one surgeon (D. P. T.) were randomized to receive posterior pericardiotomy or no posterior pericardiotomy (control group). Patients with unstable angina were included and left ventricular (LV) function was assessed at cardiac catheterization and defined as good (ejection fraction $>50 \%$ ), moderate (ejection fraction $30 \%$ to $50 \%$ ), or poor (ejection fraction $<30 \%$ ).

Operation. All operations were done with the use of intermittent global ischemia with the systemic tempera- ture allowed to drift to $34^{\circ} \mathrm{C}$. Cardiopulmonary bypass was established with a roller pump and nonpulsatile flow between 2.0 and $2.4 \mathrm{~L} \cdot \mathrm{m} \cdot \min$. A Cobe CML membrane oxygenator and alpha-stat arterial carbon dioxide tension management were used and arterial pressure maintained at 50 to $80 \mathrm{~mm} \mathrm{Hg}$.

Pericardiotomy. Patients randomized to the pericardiotomy group received a $4 \mathrm{~cm}$ posterior pericardial incision below the left inferior pulmonary vein parallel and posterior to the phrenic nerve as described by Mulay and colleagues. ${ }^{3}$

Drainage. Anterior mediastinal and left pleural drains were routinely placed with suction at $10 \mathrm{~mm} \mathrm{Hg}$. Hourly blood loss was recorded until drain removal the following day.

Postoperative electrocardiography. The electrocardiogram (ECG) was monitored continuously for the first 48 hours after operation and subsequently by 12-lead ECGs as required. In the event of SVT the serum potassium 
level was corrected if less than $4.5 \mathrm{mmol} / \mathrm{L}$. If the SVT persisted sotalol or digoxin administration was begun depending on LV function.

Statistics. The analysis of the data was done with SPSS software. Clinical data are expressed as the mean plus or minus the standard deviation. Differences were analyzed with a $t$ test or a Mann-Whitney test for nonparametric data. The differences were considered to be significant for $p$ values $<0.05$.

Results. The groups were well matched with regard to age, sex, medications received (particularly beta blockers), LV function, and unstable angina necessitating administration of intravenous heparin and nitrates (Table I). The groups were similar with respect to the number of grafts (including the use of internal thoracic and radial artery grafts), ischemic time, and cardiopulmonary bypass time (Table I).

Postoperative factors. There was one hospital death in each group in patients with severely impaired LV function. One patient underwent reoperation because of bleeding from a side branch of the internal thoracic artery. One patient in each group required insertion of an intraaortic balloon pump but both patients recovered and were discharged from the hospital. The groups were similar with regard to duration of mechanical ventilation.

Incidence of SVT. There was no significant difference between the two groups with respect to the incidence of SVT or AF, which occurred in 13 patients $(26 \%)$ in the pericardiotomy group and in 10 patients $(20 \%)$ in the control group (Table II). In the two groups the combined incidence of SVT was 5 patients of 45 patients younger than 60 years old and 18 of 55 patients older than 60 years $(p<0.01)$.

Chest drainage. The fenestration group had significantly greater chest drainage at 12 hours $(831 \pm 303 \mathrm{ml}$ vs $660 \pm$ $238 \mathrm{ml})$ and in total $(1032 \pm 367 \mathrm{ml}$ vs $813 \pm 282 \mathrm{ml})$ compared with values in the control group (Table II).

Discussion. The cause of postoperative SVT is not entirely clear. In a study of 1666 patients undergoing isolated $\mathrm{CABG}$ the overall incidence of $\mathrm{AF}$ was $28 \%$, with the major occurrence 2 days after the operation. ${ }^{4}$ Univariate and multivariate analyses identified increased patient age to be the dominant factor promoting AF and multivariate analysis showed that postoperative beta-blocker therapy conveyed considerable protection against AF $(p=0.001)$ but was less effective in older patients. ${ }^{4}$

Pericardial effusion has also been implicated in the development of $\mathrm{AF}^{2}$ and Mulay and colleagues ${ }^{3}$ reported a dramatic reduction in the incidence of pericardial effusions and SVT with a posterior pericardial incision. Their findings were, however, weakened by nonrandomization of the patients and failure to describe either the number of surgeons involved or the method or methods of myocardial protection used.

In our study prospective randomization and the fact that all operations were done by one surgeon resulted in two similar groups with respect to preoperative, intraoperative, and postoperative factors. The incidence of SVT was not statistically different in the two groups and occurred in 10 patients (20\%) in the control group and 13 $(26 \%)$ in the fenestration group. The overall incidence of SVT of $23 \%$ is similar to the $19 \%$ incidence previously reported from this unit also with the use of intermittent global ischemia. ${ }^{5}$ The observation that increased age may be the most important factor for the development of $\mathrm{AF}$ is supported in the current study. In the two groups combined the incidence of SVT was 5 patients of 45 patients younger than 60 years old and 18 of 55 patients older than 60 years $(p<0.01)$.

Our study demonstrated a significantly greater pleural drainage in the posterior pericardiotomy group both at 12 hours and in total, which implies that this does result in more effective pericardial drainage but does not reduce the incidence of AF. It is unlikely that increased drainage was the result of bleeding from the pericardial incision because the edges were cauterized and specifically checked for bleeding.

There are two potential limitations of our study. First, we did not perform continuous ECG monitoring after 48 hours unless there was clinical suspicion of cardiac arrhythmia. The aim of our study was, however, only to detect persistent "clinically relevant" AF that would necessitate medication. Second, we did not perform echocardiography to quantify the incidence or size of any pericardial effusions. Mulay and colleagues ${ }^{3}$ used echocardiography to confirm pericardial effusions in $8 \%$ of patients with a posterior pericardiotomy and in $40 \%$ of control patients $(p<0.001)$ and concluded that pericardiotomy reduced the incidence of pericardial effusions and SVT. Although the increased chest drainage in the posterior pericardiotomy group confirms that this maneuver results in more effective pericardial drainage, posterior pericardiotomy does not reduce the incidence of SVT and is not associated with any other difference in outcome. Unless clinical benefit of improved pericardial drainage is confirmed the routine performance of posterior pericardiotomy cannot be recommended.

\section{REFERENCES}

1. Pires LA, Wagshal AB, Lancey R, Huang SK. Arrhythmias and conduction disturbances after coronary artery bypass grafting surgery: epidemiology, management, and prognosis [Review]. Am Heart J 1995;129:799-808.

2. Angelini GD, Penny WJ, El-Ghamary F, et al. The incidence and significance of early pericardial effusion after open heart surgery. Eur J Cardiothorac Surg 1987;1:165-8.

3. Mulay A, Kirk AJB, Angelini GD, Wisheart JD, Hutter JA. Posterior pericardiotomy reduces the incidence of supraventricular arrhythmias following coronary artery bypass surgery. Eur J Cardiothorac Surg 1995;9:150-2.

4. Fuller JA, Adams GG, Buxton B. Atrial fibrillation after coronary artery bypass grafting: is it a disorder of the elderly? J Thorac Cardiovasc Surg 1989;97:821-5.

5. Butler J, Chong JL, Rocker GM, Pillai R, Westaby S. Atrial fibrillation after coronary artery bypass grafting: a comparison of cardioplegia versus intermittent aortic cross-clamping. Eur J Cardiothorac Surg 1993;7:23-5. 